IP Periodica Polytechnica Civil Engineering

\author{
59(4), pp. 487,493, 2015 \\ DOI: $10.3311 /$ PPci.7926 \\ Creative Commons Attribution (1) \\ RESEARCH ARTICLE
}

\section{Additive Effects of Road \\ Intersection-Specific Volume-delay Functions}

\author{
Gergely Vasvári
}

Received 20-01-2015, revised 27-03-2015, accepted 30-03-2015

\begin{abstract}
Describing the relationship between vehicle volume and travel time delay on road sections consisting multiple intersections in the context of junction quantity is favorable to extend the advantage of intersection-specific volume-delay functions. This paper demonstrates a microsimulation method combined with a non-linear function fitting algorithm to determine unbiased delay function parameters and inspect the effect of junction number on time delays. Results are bifold: first the distance between intersections deemed irrelevant regarding delay function shape, second the implicit scaling property of homogenous junction chains is revealed. Consequently the definition of time delay on uniform intersection chains can be determined by a sole parameter set for the volume-delay function and the addition of a single scaling variable.
\end{abstract}

\section{Keywords}

Non-Linear Least-Squares Method · Function Fitting · GaussNewton Method · Volume-Delay Function · VISSIM

\section{Gergely Vasvári}

Department of Highway and Railway Engineering, Faculty of Civil Engineering, Budapest University of Techology and Economics, Múegyetem rkp. 3, H-1111 Budapest, Hungary

e-mail: vasvari@uvt.bme.hu

\section{Introduction}

This paper merges and extends two previously introduced concepts: intersection-specific time-delay functions [1] and affection radius of singular junctions [2].

Relationship of traffic volume and travel time delay of vehicles passing specific road intersections may be described with a formula analogous to volume-delay functions of uninterrupted road sections [1]. Presumably the number of, and distance between junctions on a road section also has large impact on the shape delay functions besides the intersection layout. Of these two additional aspects the effect of intersection quantity is evident: the more junctions are on a road, the higher is the delay of individual vehicles. The current paper presents a specific method to evaluate traffic flow measurements and determine intersection-specific volume-delay function parameters for different types of homogenous junction-chains on a closed road section.

After laying down the principles of function fitting and before switching over to analyse the effect of junction quantity, assessment of the second aspect will be presented. Distance between particular intersections is also an important attribute in the quantization of time delay. When junctions are close, congestion caused by an oversaturated intersection is affecting neighbouring junctions' traffic flow triggering traffic jams remotely or amplifying already existing ones. For the spatial limit of junction interaction the concept of 'affection radius' [2] is utilised in the paper to demonstrate that intersection distance has no effect on the shape of delay function - due to a specific restriction of function fitting.

\section{Framework}

Instead of road side measurements a number of microsimulations were employed to provide detailed traffic flow metrics as they replicate real-life driving behaviour and traffic situations adequately [3]. The possibility to maintain a controlled input volume of vehicles throughout the analysed network segment makes data separation and function fitting a straightforward task compared to using real life measurements.

Simulation models created for previous analysis [1] were 
stringed together to create chains of two and three intersections. To clearly discern junction-specific details of superposition the investigation was restricted to homogenous intersection sets. Microsimulation models of the experiment share the following features:

- Due to their higher stability and cleaner separation of results, all model junctions had two through lanes in both directions.

- Every model junction had a separate left-turning lane on the major road, and no turning lanes on the minor road

- Heavy vehicle ratio was confined to $10 \%$

- Minor roads had vehicle inputs of $200 \mathrm{v} / \mathrm{h}$

- Turning ratios were defined per Table 1 . Note that every approach of the major road has only two directions at three-leg junctions: either 'left and through' or 'right and through'. Percentages should be interpreted accordingly (i.e. $90 \%+10 \%)$

Tab. 1. Turning ratios

\begin{tabular}{|c|c|c|c|c|c|}
\hline & \multicolumn{3}{|c|}{ 3-leg } & \multicolumn{2}{|c|}{ 4-leg } \\
\hline & \multicolumn{2}{|c|}{ major } & \multirow{2}{*}{ minor } & \multirow{2}{*}{ major } & \multirow{2}{*}{ minor } \\
\hline & left turn & right turn & & & \\
\hline left & $10 \%$ & - & $50 \%$ & $10 \%$ & $20 \%$ \\
\hline through & $90 \%$ & $90 \%$ & - & $80 \%$ & $60 \%$ \\
\hline right & - & $10 \%$ & $50 \%$ & $10 \%$ & $20 \%$ \\
\hline
\end{tabular}

- The composition of microsimulation models from the constructs introduced earlier [1] is according to Table 2] Length of downstream road section leading to the junction chain and intermediate road sections linking particular intersections are displayed on the table. Upstream road section length was uniform $-1 \mathrm{~km}$. The notation concept used throughout this paper is: $\mathbf{n} \times \mathbf{m L}$, where $\mathbf{n}=$ 'number of intersections', and $\mathbf{m}=$ 'number of junction legs' (i.e. 3 or 4 ). A suffix of in denotes junction compositions where intersections were inside each other's affection radius (see [2] for details).

Volume-delay function parameters were set during the research for every intersection model of Table 2 according to the results of microsimulations. It must be noted that although traffic composition and junction configuration seem to be a restricted set, the following methodology can be utilized to discern parameter sets and reveal similiraties of other junction types.

\section{Function fitting}

Determining function parameters based on a set of observed traffic flow data needed a verifiable method to support unbiased comparison for the analysis of additive effects. Transportation modelling makes use of several different volume-delay functions. Comparison of a generic delay function curve to real life traffic flow observations is displayed on Fig. 1. Taking into account the deterministic nature of modelling algorithms a realistic delay curve is amended at the point of capacity to enable explicit definition of delay and also enable convergence during traffic assignment. Therefore when a delay function is to be used in transportation network models (e.g. with VISUM, EMME CUBE etc. software) the curve should be fitted exclusively to uncongested measurement data.

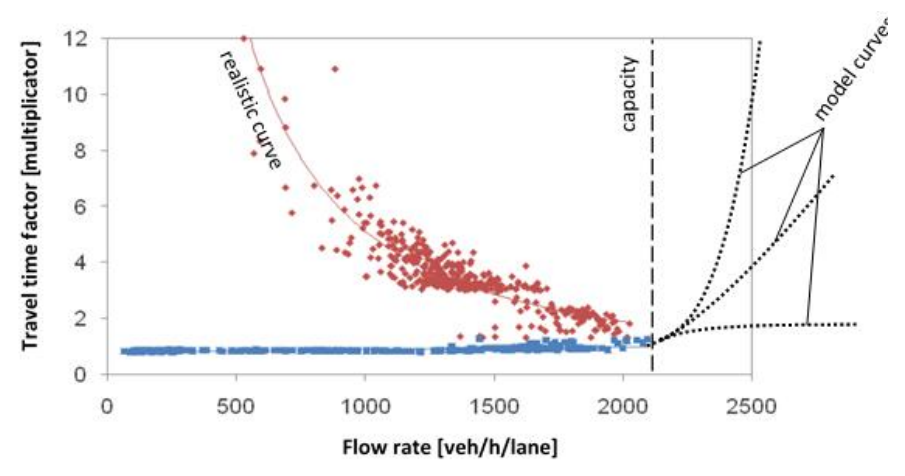

Fig. 1. VDF curves

Amongst the numerous volume-delay formulae (e.g. [4-7]]) the function of choice for the current research was the 'Conical function' [5]. The original formula was modified by modelling practitioners as displayed on Eq. (1) to give it greater flexibility.

$$
\begin{aligned}
& F(\bar{x}, t)= \\
& =x_{1}\left[x_{2}+\sqrt{x_{3}^{2}\left(x_{4}-\frac{t}{x_{5}}\right)^{2}+\left(\frac{2 x_{3}-1}{2 x_{3}-2}\right)^{2}}-\right. \\
& \left.-x_{3}\left(x_{4}-\frac{t}{x_{5}}\right)-\frac{2 x_{3}-1}{2 x_{3}-2}\right]
\end{aligned}
$$

Where vector $\bar{x}$ represent the function parameters, and $t$ the function variable (traffic volume).

The composition of parameters were analysed and modified during the research to better support convergence of the fitting algorithm (see below). It must also be noted that the following methodology is suitable for other types of volume-delay functions as well.

A common solution for the problem of multi-parameter function fitting is through the use of Non-Linear Least-Squares Method (NLLSM) where the task is to minimize the sum of squares of residuals: The minimization problem is usually solved with an iterative method (gradient descent, conjugate gradient, line search etc.). For the current research a modified Gauss-Newton method was implemented which could be regarded as a specific type of gradient-descent method. Formal definition and solution of the problem may be found in [8]. Specific derivatives constructing the Jacobian matrix will be presented in the following paragraphs.

\subsection{Reduction of parameters}

Number of unknown elements - delay function parameters at present - significantly affects iteration complexity thus effi- 
Tab. 2. Microsimulation model compositions

\begin{tabular}{cccc}
\hline notation & junctions & downstream rd. sct. & intermediate rd. sct. \\
\hline $1 \times 3 \mathrm{~L}$ & One 3-leg & $5 \mathrm{~km}$ & - \\
$2 \times 3 \mathrm{~L}$ & Two 3-leg & $5 \mathrm{~km}$ & $5 \mathrm{~km}$ \\
$3 \times 3 \mathrm{~L}$ & Three 3-leg & $5 \mathrm{~km}$ & $5 \mathrm{~km}$ \\
$1 \times 4 \mathrm{~L}$ & One 4-leg & $3 \mathrm{~km}$ & - \\
$2 \times 4 \mathrm{~L}$ & Two 4-leg & $3 \mathrm{~km}$ & $3 \mathrm{~km}$ \\
$3 \times 4 \mathrm{~L}$ & Three 4-leg & $3 \mathrm{~km}$ & $3 \mathrm{~km}$ \\
$2 \times 3 \mathrm{~L}$ in & Two 3-leg, affected & $3 \mathrm{~km}$ & $3 \mathrm{~km}$ \\
$3 \times 3 \mathrm{~L}$ in & Three 3-leg, affected & $3 \mathrm{~km}$ & $3 \mathrm{~km}$ \\
$2 \times 4 \mathrm{~L}$ in & Two 4-leg, affected & $1 \mathrm{~km}$ & $1 \mathrm{~km}$ \\
$3 \times 4 \mathrm{~L}$ in & Three 4-leg, affected & $1 \mathrm{~km}$ & $1 \mathrm{~km}$ \\
\hline
\end{tabular}

ciency and convergence speed of an algorithm. It is favourable to check the model function (1) for possible reduction of its parameters. These can be reduced by merging parameters $x_{3}, x_{4}$ and $x_{5}$ as shown in Eq. (2).

$$
x_{3}\left(x_{4}-\frac{t}{x_{5}}\right)=\frac{x_{5}}{x_{5}} x_{3}\left(x_{4}-\frac{t}{x_{5}}\right)=\frac{x_{3}}{x_{5}}\left(x_{4} x_{5}-t\right)
$$

Hence using substitutions of (3) the number of function parameters can be reduced.

$$
\breve{x}_{3}=\frac{x_{3}}{x_{5}} \quad \text { and } \quad \breve{x}_{4}=x_{4} \cdot x_{5}
$$

Re-numerating the parameters, the formula of the volume delay function to be fitted is (4).

$$
\begin{aligned}
& F(\bar{x}, t)=x_{1}\left[x_{2}+\sqrt{\breve{x}_{3}^{2}\left(\breve{x}_{4}-t\right)^{2}+\left(\frac{2 x_{3}-1}{2 x_{3}-2}\right)^{2}}-\right. \\
& \left.-\breve{x}_{3}\left(\breve{x}_{4}-t\right)-\frac{2 x_{3}-1}{2 x_{3}-2}\right]
\end{aligned}
$$

Further on the accent breve is omitted from variables $\breve{x}_{3}$ and $\breve{x}_{4}$ for convenience.

\subsection{Balance of sensitivity}

When function value change disproportionately by the same amount of difference in its parameters, minimization convergence cannot be assured. Using One-Factor-At-a-Time and scatterplot analyses to assess parameter sensitivity it was found that the model formula is ill-conditioned due to the highly different sensitivity in terms of its parameters. This property prevents convergence of the minimum search algorithm, thus the formula had to be modified.

Reconditioning the formula by scaling the parameters with vector $\bar{a}=\left(10^{-2} 10^{-2} 10^{-5} 1\right)^{T}$ and using the pointwise product $\bar{a} \circ \bar{x}$ instead of $\bar{x}$ the model function sensitivity toward its parameters can be equalized. Equation (5) introduces the modified formula.

$$
\begin{aligned}
& F(\bar{x}, t)=a_{1} x_{1}\left[a_{2} x_{2}+\right. \\
& +\sqrt{\left(a_{3} x_{3}\right)^{2}\left(a_{4} x_{4}-t\right)^{2}+\left(\frac{2 a_{3} x_{3}-1}{2 a_{3} x_{3}-2}\right)^{2}}- \\
& \left.-a_{3} x_{3}\left(a_{4} x_{4}-t\right)-\frac{2 a_{3} x_{3}-1}{2 a_{3} x_{3}-2}\right]
\end{aligned}
$$

\subsection{Jacobian derivatives}

The implemented minimum search algorithm requires the calculation of Jacobian matrix members. Using the preconditioned formula (5), following equations (6), (7), (8) and (9) display partial derivatives of the volume-delay function.

$$
\begin{aligned}
& \frac{\partial F(\bar{x}, t)}{\partial x_{1}}=a_{1}\left[a_{2} x_{2}+\right. \\
& +\sqrt{\left(a_{3} x_{3}\right)^{2}\left(a_{4} x_{4}-t\right)^{2}+\left(\frac{2 a_{3} x_{3}-1}{2 a_{3} x_{3}-2}\right)^{2}}- \\
& \left.-a_{3} x_{3}\left(a_{4} x_{4}-t\right)-\frac{2 a_{3} x_{3}-1}{2 a_{3} x_{3}-2}\right]
\end{aligned}
$$

$$
\frac{\partial F(\bar{x}, t)}{\partial x_{2}}=a_{1} x_{1} a_{2}
$$

$$
\begin{aligned}
& \frac{\partial F(\bar{x}, t)}{\partial x_{3}}= \\
& =a_{1} x_{1}\left[\frac{a_{3}^{2} x_{3}\left(a_{4} x_{4}-t\right)^{2}-\frac{4 a_{3}^{2} x_{3}-2 a_{3}}{\left(2 a_{3} x_{3}-2\right)^{3}}}{\sqrt{\left(a_{3} x_{3}\right)^{2}\left(a_{4} x_{4}-t\right)^{2}+\left(\frac{2 \cdot x_{3}-1}{2 \cdot x_{3}-2}\right)^{2}}}-a_{3} a_{4} x_{4}+\right. \\
& \left.+a_{3} t+\frac{2 a_{3}}{\left(2 a_{3} x_{3}-2\right)^{2}}\right]
\end{aligned}
$$

$$
\begin{aligned}
& \frac{\partial F(\bar{x}, t)}{\partial x_{4}}= \\
& =a_{1} x_{1}\left[\frac{\left(a_{3} x_{3}\right)^{2}\left(a_{4} x_{4}-t\right)}{\sqrt{\left(a_{3} x_{3}\right)^{2}\left(a_{4} x_{4}-t\right)^{2}+\left(\frac{2 a_{3} x_{3}-1}{2 a_{3} x_{3}-2}\right)^{2}}}-a_{3} x_{3} a_{4}\right]
\end{aligned}
$$




\subsection{Fitting considerations}

Another detail has to be considered when using the NLLSM for VDF parameterization. As it was mentioned above, fitting to congested data has to be avoided by the removal of unnecessary measurements from vectors $\bar{t}$ and $\bar{f}$. In the developed software an adjustable upper cap toward accepted time delay was implemented to account for this need.

Observation showed that success of fitting mainly depend on the initial value of parameter $x_{4}$ - provided that upper cap was set to a proper value (Fig. 2). This parameter has major effect on the horizontal position of the vertical asymptote and has to be set to a value where the asymptote is between the ordinate and the congested part of the observed data.

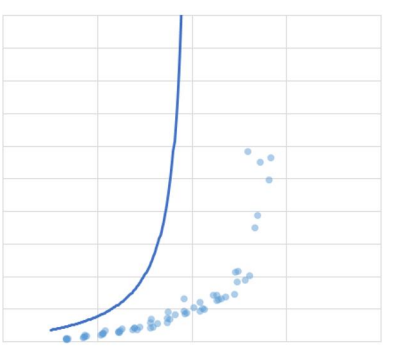

volume $[\mathrm{veh} / \mathrm{h}]$

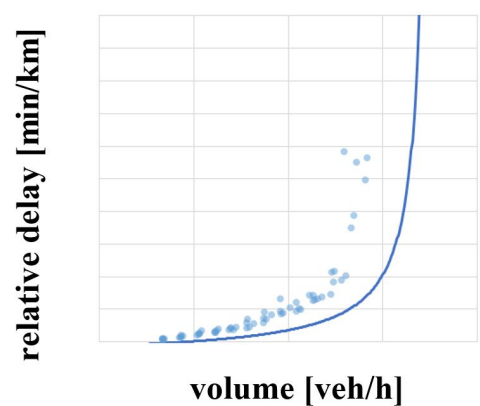

volume [veh/h]
Fig. 2. Examples of adequate (left) and unsuitable (right) initial values of $x_{4}$

\section{Evaluation}

Although delay measurements of microsimulation runs were collected on a 15-minute (simulation time) basis to provide adequate sampling for analysis if necessary, functions were fitted first to aggregated data (hourly sum of vehicles and average of delay). This method was found suitable for further analysis. The main reason to use a reduced data set in the NLLSM was to avoid calculations with oversized vectors and matrices.

Different model setups yield to different travel distance for the vehicles. To have comparable data, delay measurements were recalculated to minute $/ \mathrm{km}$ units to determine the relation of junction quantity and delay function formula.

\subsection{Context of affection radius}

Time delay measurements of simulation runs showed identical results to traffic flow theories' fundamental speed-volume diagram. Considering that speed and travel time are parallel notions and time delay is linear consequence of travel time these results are sound. Vehicles traversing junctions which are inside the affection radius acquire more time delay than vehicles on more spacious junction chains, but the difference is restricted to the congested state. Below the point of theoretical capacity, model setups show similar time delays regardless of intersection placement. This behaviour is illustrated on the following figures: the 'full range' of measurements show every collected data point, and dissimilarity between congested states are apparent (Fig. 3). Time delay measurements of junctions within the affection radius are higher than of unaffected junctions' as it is expected.

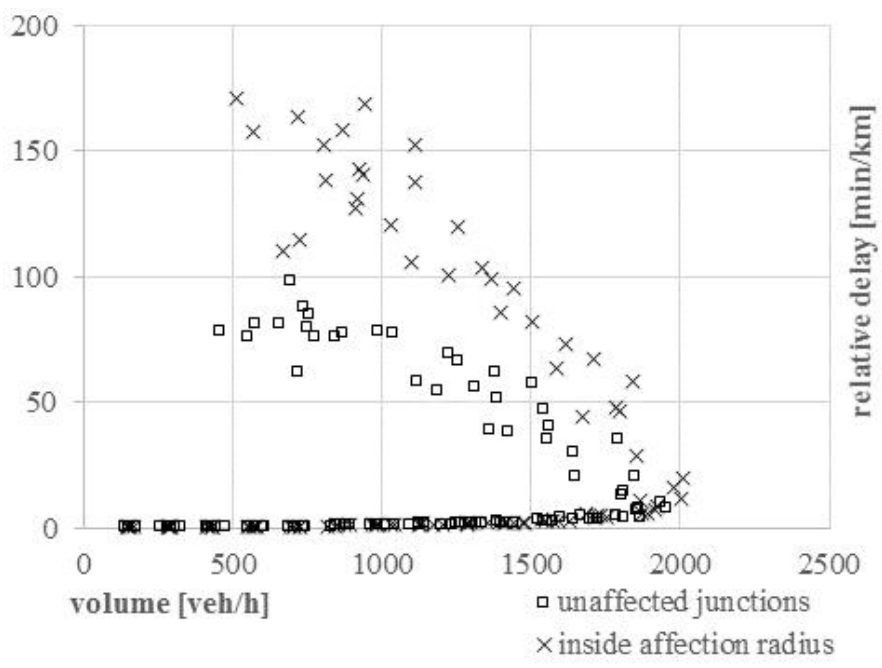

Fig. 3. Time delay measurements of two 3-leg junction (full range)

However inspection on the lower ranges of time delay reveals the close similarity of uncongested state (Fig. 4). Comparing 3-leg and 4-leg (not displayed here) junction chains, the effects match, although the lower throughput of the 4-leg variant is obvious - due to the higher number of vehicle conflicts at the intersections.

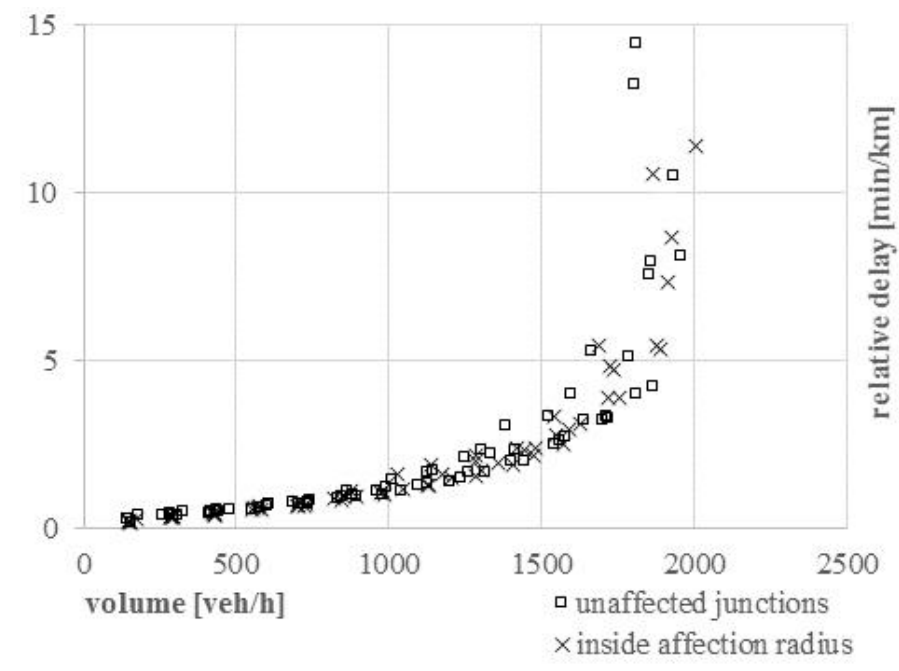

Fig. 4. Time delay measurements of two 3-leg junction (uncongested range)

As it was discussed above volume-delay functions have to be fitted on uncongested traffic data: it is necessary to employ a model curve past capacity to have a one-to-one mapping in the function. Consequently the distinction of road sections based on junction distance is not needed. To have numeric evidence Fig. 5 shows a function fit performed on two data sets (junctions inside and outside of the affection radius), and also gives parametric details of the result. Difference between the two function curves are limited to the 'model curve' section, which part was omitted from the fitted dataset. Concerning relevant measurements, the determined parameters are very similar. 


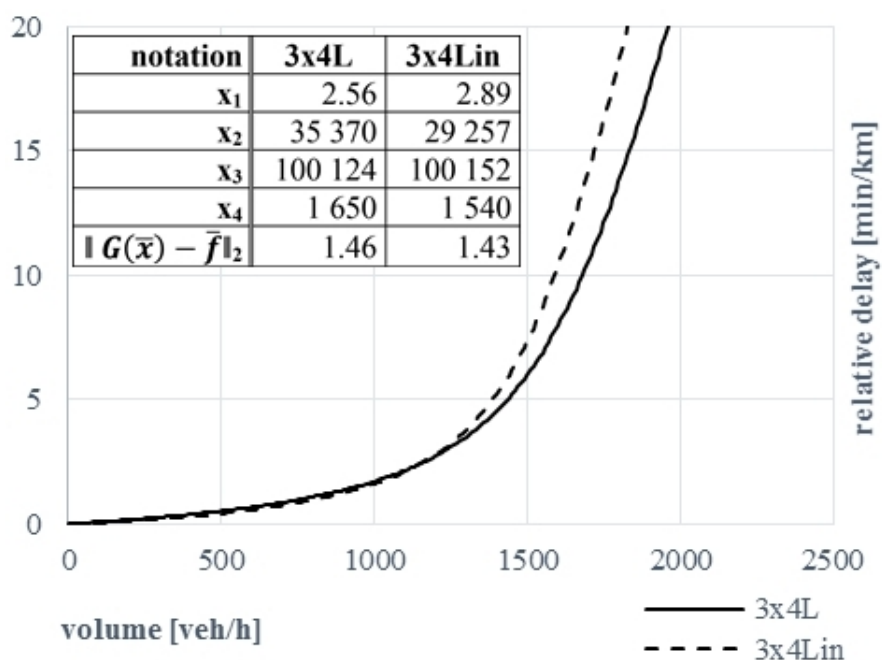

Fig. 5. Fitted VDF curves with function parameters

\subsection{Context of intersection quantity}

Time delays are specific to junction type (3-leg or 4-leg) and also depending on the number of intersections on the road. By making use of the Non-Linear Least-Squares Method (NLLSM) mentioned earlier, estimation of delay function parameters was realized (Table 3). The Euclidian norm $\|g\|_{2}$ provides goodness of fit metrics.

\section{Analysis}

Observation of the parameters tell that variations are in similar order of magnitude, and - excluding $x_{1}$ and $x_{2}$ - each form a tight numeric range but none of them are identical. Using a specific set of parameters for every road section would require a lot of resources (time and manpower) during modelling, hence it is worth to investigate whether the formulation can be unified. By taking a look at the function graphs it is clear without further calculations that one VDF could not be reproduced by simple scaling of one of the other curves. However the degree of deviation could be better assessed. Fig. 6 shows 3-leg junction VDFs (black curves) and additionally the ratio of specific functions, marked with grey, defining function ratios as 10 .

$$
R_{t y p 1, t y p 2}(t)=\frac{F\left(\bar{x}_{t y p 1}, t\right)}{F\left(\bar{x}_{t y p 2}, t\right)}
$$

where typ1, typ 2 are examined junction types (e.g. $2 \times 3 \mathrm{~L}$ and $1 \times 3 \mathrm{~L})$ and indexed vectors of $x$ are the set of corresponding function parameters.

Dividing the function values of junction chains by the function values of the singular intersection shows a quasi-linear relationship up to the point where traffic volumes exceed the capacity of the model setup at one of the functions compared. After that point, ratios show similarities to their own types but the effect is exaggerated by the increasing number of junctions. There are some deviations to this logic, mostly apparent at 4-leg junctions (not displayed here), where the fitted curve of the singular junction is irregular at very low traffic volumes, producing a large spike when using VDF values as a denominator. This irregularity can be explained with the mechanisms of the Least Squares Method: the overweighing properties of the method at high-gradient parts of the function, meaning that low gradient parts are not fitted as accurately as high gradient parts. This attribute produces the discrepancy between different VDFs around zero traffic volume values.

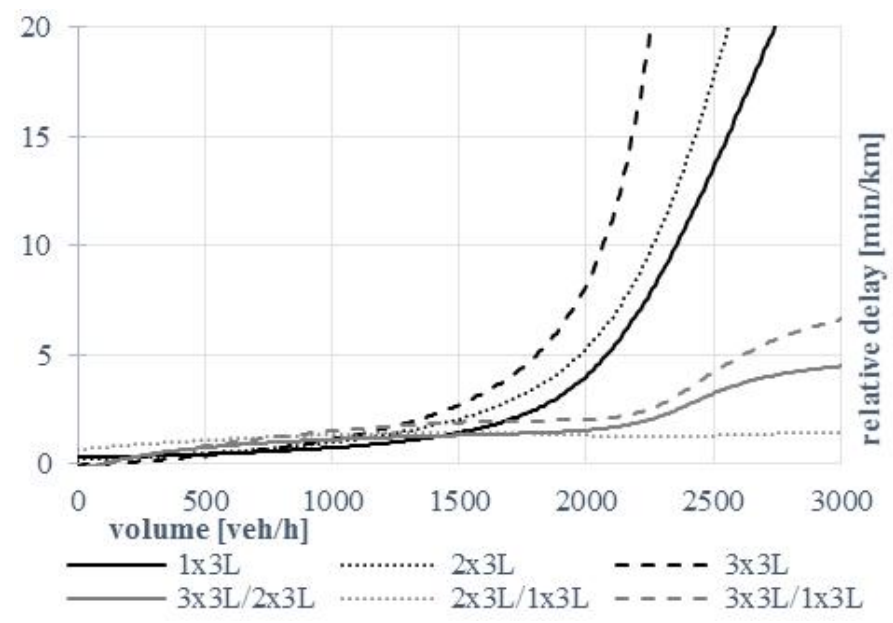

Fig. 6. Delay curves and ratios of 3-leg junction and junction chains (Grey curves show the ratio of different VDF values)

Although this inspection did not give first hand results, it showed that while the functions themselves diverge, there is some similarity in their behaviour. This confirmation of the hypothesis leads to another inquiry: a similar examination of the inverse functions. Considering the complexity of formula (5) and the fact that inverse functions are not needed in other parts of the research, inverses were constructed by numeric goal-seeking in MS Excel at specific $f(t)$ function values.

Analytically the function inverse can be written as 11 .

$$
F^{i n v}(\bar{x}, f)=T(\bar{x}, f)
$$

Then inverse function ratio as (12).

$$
I_{t y p 1, t y p 2}(f)=\frac{T\left(\bar{x}_{t y p 1}, f\right)}{T\left(\bar{x}_{t y p 2}, f\right)}
$$

where $f$ represent the variable along the ordinate.

By executing the division, resulting graphs (Fig. 7 prove to be close to linear - even constant - indeed.

Again, there are irregularities at low function values due to the inaccuracy of fitting. Overlooking the slight curve of these graphs, approximate scaling factors can be determined for the function variable, hence it is possible to describe all three delay functions with a single parameter set and the additional scaling factor. Results of the parameter substitution are on Fig. 8 ,

Here separate delay functions are displayed in black using separate parameter sets determined by NLLSM, along with new curves using a single (unified) formula and a scaling factor displayed in grey. Note that the unified function curve $(1 \times 3 \mathrm{~L}$ 
Tab. 3. Function parameters determined by NLLSM

\begin{tabular}{ccccccc}
\hline notation & $\mathbf{1} \mathbf{x} \mathbf{~ L}$ & $\mathbf{2 \times 3 L}$ & $\mathbf{3 \times 3 L}$ & $\mathbf{1 \times 4 L}$ & $\mathbf{2} \mathbf{x} \mathbf{4}$ & $\mathbf{3 \times 4 L}$ \\
\hline $\boldsymbol{x}_{\mathbf{1}}$ & 1.50 & 2.68 & 13.57 & 1.79 & 1.32 & 5.53 \\
$\boldsymbol{x}_{\mathbf{2}}$ & 37308 & 39868 & 22499 & 63094 & 60710 & 35935 \\
$\boldsymbol{x}_{\mathbf{3}}$ & 100128 & 100116 & 100211 & 100067 & 100069 & 100127 \\
$\boldsymbol{x}_{\mathbf{4}}$ & 2123 & 2287 & 2349 & 2591 & 2144 & 2189 \\
$\|\mathbf{g}\|_{\mathbf{2}}$ & 6.07 & 2.48 & 4.62 & 4.17 & 1.10 & 0.82 \\
\hline
\end{tabular}

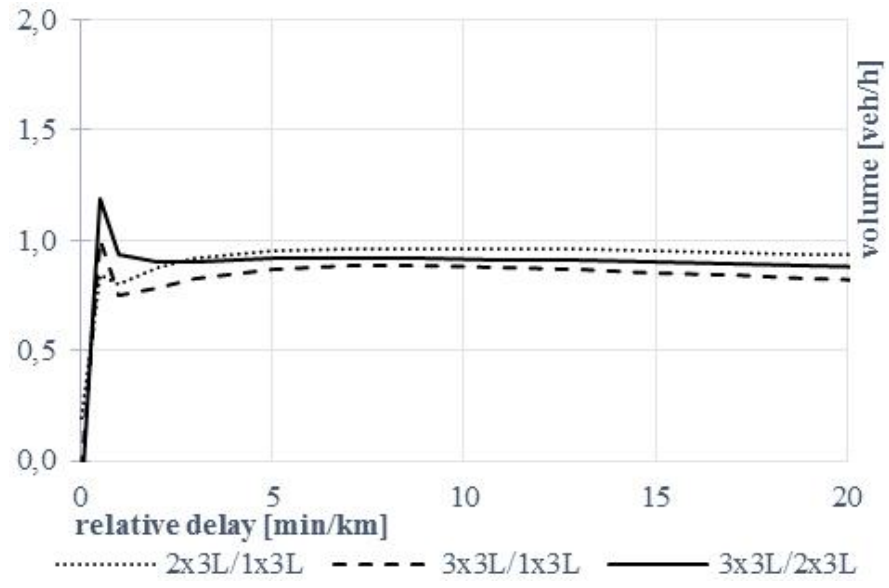

Fig. 7. Ratios of 3-leg junctions' inverse VDFs

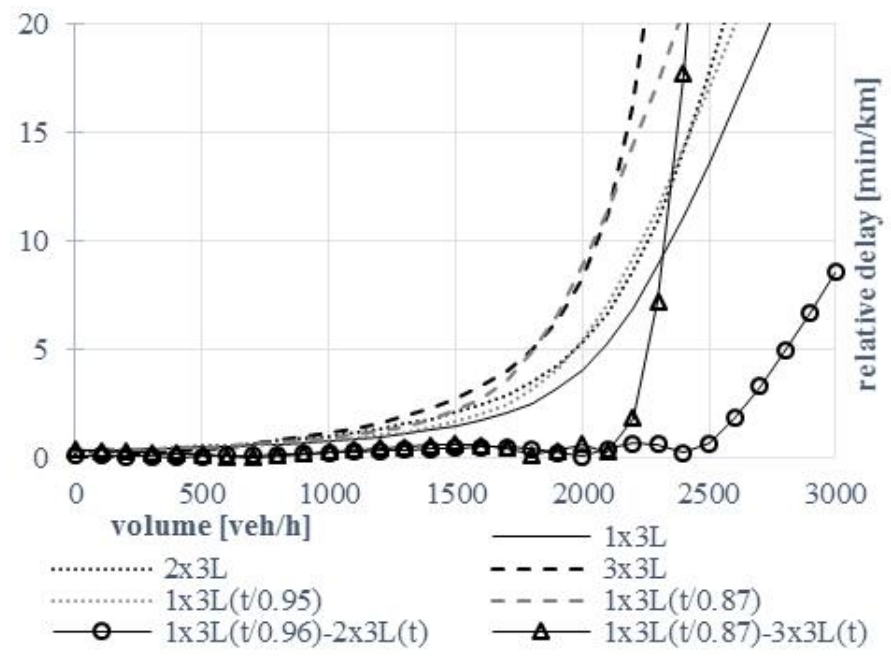

Fig. 8. Unified VDF for 3-leg junctions, based on 1 x $3 \mathrm{~L}$ formula

variant) is not repeated on the chart. To help comparison, differences between fitted and unified formulae are also presented on the figure - using circular and triangular markers. The conspicuously large discrepancy at higher traffic volumes could be deemed less significant as it is on the 'model' part of the curve, where measured data had little to no impact on the function shape. Aside from this 'model' part of the functions, difference between firsthand fitted curves and ones determined by a unified formula are very low. Table 4 lists the parameters and scaling factor $b$ of the unified formula for comparison with fitted parameters on Table 3 The low value of Euclidian norms $\left(\|g\|_{2}\right)$ prove the concept of substitution successful.

However using the VDF of the singular junction model propagates the inaccuracy of fitting at lower volumes to other intersec-
Tab. 4. Fitted and redefined function parameters

\begin{tabular}{ccc}
\hline notation & $\mathbf{1} \mathbf{x}$ 3L unified & $\mathbf{3} \mathbf{x}$ 3L unified \\
\hline $\boldsymbol{x}_{\mathbf{1}}$ & 2,68 & 2,68 \\
$\boldsymbol{x}_{\mathbf{2}}$ & 39868 & 39868 \\
$\boldsymbol{x}_{\mathbf{3}}$ & 100116 & 100116 \\
$\boldsymbol{x}_{\mathbf{4}}$ & 2287 & 2287 \\
$\boldsymbol{b}$ & 0.95 & 1.10 \\
$\|\mathbf{g}\|_{\mathbf{2}}$ & 3.80 & 3.50 \\
\hline
\end{tabular}

tion chains. A better curve shape can be derived from the twojunction variant It is also a viable solution to manually adjust the curve shape and use it as a basis for all three VDFs. Function differences also showed improvement - although marginal - using the two-junction variant as a basis for unified formulae.

Four leg junction delays can also be analysed in the same manner as three leg junctions: first determining the inversefunction ratios, then using the results and replace the three parameter set with a single one and an additional scaling factor.

\section{Conclusions}

Implementation of the Non-Linear Least-Squares Method was successful. Supporting convergence, a set of scaling factors were incorporated into Eq. (4) resulting in Eq. (5). These factors may be omitted later, using the pointwise product $\bar{a} \circ \bar{x}$ directly in the original equation.

Microsimulation results showed that in the region of uncongested traffic flow it is not necessary to distinguish upon intersection distance. Simulations also confirmed the different behaviour of affected and unaffected junctions under congested traffic.

Scaling of the function variable successfully unifies the VDF formula of homogenous junction series for each intersection type. The operation eliminates the need of separate parameter sets for different number of intersections on the link but introduces a new factor $(b)$ to the formula, expanding (4) to (13).

$$
\begin{aligned}
& F(\bar{x}, t)=x_{1}\left[x_{2}+\sqrt{x_{3}^{2}\left(x_{4}-b \cdot t\right)^{2}+\left(\frac{2 x_{3}-1}{2 x_{3}-2}\right)^{2}}-\right. \\
& \left.-x_{3}\left(x_{4}-b \cdot t\right)-\frac{2 x_{3}-1}{2 x_{3}-2}\right]
\end{aligned}
$$

\section{Further uses}

The demonstrated methodology can be applied to chains of other junction types to determine specific delay function param- 
eters. Delays should also be analysed in the context of heavy vehicle ratio and minor flow volumes. The inspection of mixed intersection chains is a straightforward extension of the research project.

\section{References}

1 Vasvari G, Volume-delay functions of minor junctions created by microsimulation, Pollack Periodica, 9(1), (2014), 29-40, DOI 10.1556/Pollack.9.2014.1.4

2 Vasvari G, Affection radii of congested junctions on traffic networks, Periodica Polytechnica Civil Engineering, 58(1), (2014), 87-92, DOI 10.3311/PPci.7409

3 Fellendorf M, Vortisch P, Validation of the Microscopic Traffic Flow Model VISSIM in Different Real-World Situations, TRB, 2001.

4 Bureau of Public Roads, Traffic Assignment Manual, US Dept. of Commerce, Urban Planning Division, 1964.

5 Spiess H, Conical Volume-Delay Functions, Transportation Science, 24(2), (1990), 153-158, DOI 10.1287/trsc.24.2.153

6 Akcelik R, Travel time functions for tranport planning purposes: Davidson's function, its time-dependent form and an alternative travel time function, Australian Road Research Board, 1991.

7 Jastrzebski WP, Volume Delay Functions, 2000. 15th International EMME/2 Users' Group Conference.

8 Gisbert S, Numerikus Matematika - Mérnököknek és programozóknak, Hungarian, Typotex, 2007. (in Hungarian). 